\title{
Auswirkungen von Analogiemodellen auf den Aufbau konzeptuellen Wissens im Sachunterricht der Grundschule - Beispiel Stromkreis
}

\author{
Michael Haider (iD - Maria Fölling-Albers
}

Online publiziert: 1. Juli 2020

(C) Der/die Autor(en) 2020

Zusammenfassung Naturwissenschaftlicher Unterricht bedarf vielfach konkreter Anschauungsmittel, weil Lerninhalte abstrakt und/oder aufgrund spezifischer Merkmale nicht direkt erfahrbar sind. Durch Analogiemodelle können solche Konkretisierungen erreicht werden. In der vorliegenden Studie wurde mit einem quasi-experimentellen Prä-, Post- und Follow-up-Design untersucht, inwiefern bei Schülerinnen und Schülern der dritten Jahrgangsstufe $(N=254)$ der Einsatz unterschiedlicher Analogiemodelle (Wassermodelle vs. Riemenmodelle) den Aufbau konzeptuellen Wissens zum Thema Strom (Stromfluss, Stromverbrauch (Der Begriff Stromverbrauch wird zwar im Alltag verwendet, ist aber sachlich inkorrekt. Wegen einer besseren Lesbarkeit wird auf Anführungszeichen verzichtet.)) unterstützt. Die Befunde zeigen, dass der Inhalt Stromfluss auch ohne den Einsatz von Analogiemodellen erfolgreich erarbeitet werden kann. Beim komplexeren Schwerpunkt Stromverbrauch erwies sich der Einsatz des Riemenmodells als günstiger. Differenzielle Effekte für Kinder mit ungünstigen Lernvoraussetzungen konnten nicht gefunden werden.

Schlüsselwörter Lernen mit Analogien · Konzeptaufbau · Naturwissenschaftlicher Unterricht

\footnotetext{
M. Haider $(\square) \cdot$ M. Fölling-Albers

Lehrstuhl für Pädagogik (Grundschulpädagogik), Universität Regensburg, 93040 Regensburg,

Deutschland

E-Mail: michael.haider@ur.de

M. Fölling-Albers

E-Mail: maria.foelling-albers@ur.de
} 


\title{
Use of analogy models for buildup conceptual knowledge in science education in primary schools—using the example electrical current
}

\begin{abstract}
Science education often requires concrete visual aids because abstract learning content and/or specific characteristics cannot be experienced directly. For this reason, a study with a quasi-experimental pre-, post- and follow-up design examined to what extent conceptual knowledge on the topic of electricity ("electricity flow", "electricity consumption") can be built up more successfully through the use of different analogy models (VS1 water models, VS2 belt models) compared to instruction without such models (VG). The study was carried out in third-grade classrooms $(N=254)$ where this subject is part of the curriculum. Our analyses revealed that students successfully acquired knowledge on the content of "electricity flow" without the use of analogy models. When focusing on the consumption of power it turns out that using the belt model is way more efficient. Differential effects for children with unfavorable learning conditions could not be found.
\end{abstract}

Keywords Learning with analogies $\cdot$ Concept building $\cdot$ Science education

\section{Einleitung}

Lernprozesse im Bereich der naturwissenschaftlichen Bildung bedürfen vielfach besonderer Unterstützungsmaßnahmen, denn häufig erschweren vorhandene Alltagsvorstellungen den Aufbau wissenschaftlich anschlussfähiger Konzepte. Dies betrifft zum Beispiel das konzeptuelle Wissen zum Stromkreis: So ist der Stromfluss (flieBende Ladungsträger) visuell nicht wahrnehmbar, und er erscheint auch als Kreislauf nicht plausibel (vgl. z. B. Kircher et al. 2009). Zudem kennen Kinder aus der Alltagssprache den Terminus Stromverbrauch, was aus physikalischer Sicht zu einem Fehlkonzept führen kann. Aus didaktischer Sicht ist es eine wichtige Frage, wie anschlussfähiges konzeptuelles Wissen zum Thema Stromkreis in der Grundschule aufgebaut werden kann: Zum einen ist das Thema in dieser Schulstufe prominent (vgl. z. B. für Bayern: Staatsinstitut für Schulqualität und Bildungsforschung 2017; für Nordrhein-Westfalen: Ministerium für Schule und Weiterbildung 2012), zum anderen ist die Elektrizitätslehre ein zentraler Gegenstand des Physikunterrichts der Sekundarstufe (vgl. Kircher et al. 2009). Ein gängiges didaktisches Mittel, solch abstrakten Unterrichtsinhalten zu begegnen, ist die Bildung von Analogien und der unterrichtliche Einsatz von Analogiemodellen (vgl. ebd.).

Im vorliegenden Beitrag wird untersucht, inwiefern der Einsatz von Analogiemodellen dazu beitragen kann, dass wissenschaftlich nicht belastbare Vorstellungen zum Stromkreis (hier: Stromfluss, Stromverbrauch) aufgegeben und anschlussfähige Vorstellungen aufgebaut werden. Dazu werden zwei verschiedene Modelle verglichen (ein Wassermodell und ein Riemenmodell); diese bilden Oberflächen- und Strukturmerkmale des Stromkreises unterschiedlich ab. Untersucht wird zudem der Einfluss auf den Wissenserwerb bei Schülerinnen und Schülern mit unterschiedlichem Vorwissen bzw. unterschiedlicher Schulleistung, da hier differenzielle Effekte zu erwarten sind. 


\section{Theoretischer Hintergrund}

\subsection{Conceptual Change-Prozesse beim (frühen) naturwissenschaftlichen Lernen}

Lernprozesse stellen sich bei naturwissenschaftlichen Lerninhalten immer dann als besonders schwierig dar, wenn Vorstellungen, die im Alltag aufgebaut wurden und sich dort bewährt haben, aufgegeben und durch neues Wissen ersetzt werden müssen. Dabei wird davon ausgegangen, dass alte Vorstellungen nicht einfach durch das neu gelernte Wissen ersetzt werden. Vielmehr ist eine Veränderung im Sinne einer Umstrukturierung erforderlich - diese wird seit einigen Jahrzehnten mit dem Begriff conceptual change (Konzeptveränderung bzw. Konzeptwechsel) umschrieben und erforscht (vgl. Carey 1985; Posner et al. 1982; Vosniadou und Brewer 1987; Vosniadou 1994). Dabei hat sich gezeigt, dass gerade die Vorstellungen, die von den Schülerinnen und Schülern durch Alltagswahrnehmungen aufgebaut wurden, häufig sehr stabil sind, sodass sie durch Unterricht nur schwer verändert oder gar abgelegt werden können (vgl. Duit 2011; Lohrmann und Hartinger 2012). Häufig sind auf dem Weg hin zu belastbaren Konzepten auch Zwischenvorstellungen oder eine Kombination von Fehlvorstellungen mit wissenschaftsnahen Konzepten festzustellen (vgl. Treagust und Duit 2008; Vosniadou 2007). Als belastbar können solche Konzepte angesehen werden, die den wissenschaftlichen Vorstellungen entsprechen und die in verschiedenen Situationen anwendbar sind. Um insbesondere die flexible Anwendung von Wissen zu erreichen, darf sich dieses nicht nur auf Fakten beziehen; vielmehr sollte es vielfältige Verknüpfungen zwischen Wissenselementen enthalten, die auf Verstehen abzielen (Jonen et al. 2003; Wagenschein 2010). Schon frühe Ansätze der Conceptual Change Theorien (Posner et al. 1982) machen daher darauf aufmerksam, dass Wissen, das erworben werden soll, glaubhaft, verständlich und fruchtbar/anwendbar sein muss; zugleich ist eine Unzufriedenheit mit dem alten Konzept erforderlich. In einigen Studien zum naturwissenschaftlichen Lernen im Sachunterricht zeigte sich zudem, dass unterschiedliche Kinder unterschiedlich stark von Lernangeboten profitieren (z.B. Haider 2010; Möller et al. 2002). Ein zentraler Einflussfaktor ist das Vorwissen der Schülerinnen und Schüler (vgl. z. B. de Groot und Gobet 1996; Dochy et al. 2002; Möller und Müller-Kalthoff 2000). Kinder, die über höheres Vorwissen zu einem Lerngegenstand verfügen, lernen in der Regel im Unterricht mehr, solange keine individuelle Förderung geschieht.

\subsection{Analogiemodelle zur Unterstützung von Lernprozessen}

Das Erfordernis, dass neue Konzepte verständlich sein müssen, ist bei naturwissenschaftlichen Lerninhalten häufig schwer einzulösen, da viele Lerninhalte nicht direkt beobachtbar und deshalb nicht anschaulich nachvollziehbar sind, wie zum Beispiel der Elektronenfluss oder die Schallübertragung. Aus unterrichtlicher Sicht stellt sich daher die Frage, mit welchen Maßnahmen solche Lernhinhalte verständlich gemacht werden können. Ein gängiges Mittel dazu ist die Bildung von Analogien (als Herstellung von Parallelen oder Ähnlichkeiten) und der Einsatz von Analogiemodellen (für die Didaktik der Physik vgl. z. B. Kircher et al. 2009). Solche Analogiemodel- 
le sollen die Erkenntnisvermittlung unterstützen, indem sie Unterrichtsgegenstände oder Prozesse, die nicht sichtbar, zu groß, zu klein, zu langsam, zu schnell etc. sind, vorstellbar machen (vgl. ebd.). Analogien kommt im didaktischen Einsatz somit eine Brücken- (Duit und Glynn 1995) oder Mittlerfunktion (Kircher 1995) zu: Schülerinnen und Schüler greifen auf ihr Wissen in einem sekundären, anschaulichen Bereich zurück und können dadurch Bezüge (Analogien) zu dem zu erlernenden primären Lernbereich herstellen (Analogieschlüsse) (vgl. u.a. Duit und Glynn 1995; Hesse 1991; Kircher 1995; Spreckelsen 1997).

Analogien fokussieren zunächst auf sich entsprechende Begriffe bzw. Objekte und Relationen. Diese werden in einem sekundären Lernbereich veranschaulicht und dadurch nachvollziehbar gemacht. Anschließend werden die Entsprechungen zu dem primären (weniger anschaulichen) Lernbereich identifiziert (vgl. z. B. Haider 2010; Kircher et al. 2009).

Der Prozess der Analogiebildung geschieht in verschiedenen Phasen. Sternberg (1977) wies anhand einer klassischen Analogie der Form A:B=C:D Phasen in der Reihenfolge Encoding, Inference, Mapping, Application, Justification und Preparation-Response nach. Für Analogien, wie sie in der vorliegenden Studie im fachdidaktischen Sinn gedacht werden, werden nach Ruppert (2017) v. a. die Phasen Strukturieren, Abbilden (entspricht Mapping und Application), Schließen (Inference) und Beurteilen (Justification) als wichtig erachtet. Auch wenn bei Sternberg und Ruppert die Benennung der Phasen nicht in allen Begriffen übereinstimmt, so bildet in beiden Ansätzen der Mapping-Prozess das Kernstück der Analogiebildung; dabei gilt die Strukturierung der Elemente als ein wesentlicher Bestandteil des Mappings.

Die Strukturierung und der Mapping-Prozess werden in der Structure Mapping Theory (Gentner 1983) in den Fokus genommen. Die Kernaussage dieser Theorie ist, dass erfolgreiches Lernen mit Analogiebildungen insbesondere dann geschieht, wenn es gelingt, zentrale Elemente und deren Relationen in den einzelnen Repräsentationen zu identifizieren und danach erfolgreich Bezüge zwischen den verschiedenen Repräsentationen herzustellen. Solche Mapping-Prozesse können sich sowohl auf die Oberflächenstruktur (z. B. Farbe, Form, Größe) als auch auf die Tiefenstruktur (z.B. Funktionsweisen oder Gesetzmäßigkeiten) von naturwissenschaftlichen Phänomenen beziehen. Mapping-Prozesse, die v.a. die Oberfläche der Repräsentationsformen in den Blick nehmen (syntaktisches Mapping, Gentner 1983), können zwar die Aufmerksamkeit, das Interesse und die Motivation der Schülerinnen und Schüler erhöhen (vgl. u. a. Lenzner 2009); sie können aber auch zu einem erhöhten cognitive load und damit zu einer oberflächlicheren Informationsverarbeitung führen (vgl. u.a. Mayer et al. 2001). Eine strukturell tiefere Verarbeitung (semantisches Mapping, Gentner 1983) kann dagegen eine tiefere Elaboration (Lenzner 2009) auslösen. Nach Gentner ist hier eine weitere Unterscheidung zu treffen: Im Vergleich zum analogen Denken $(\mathrm{A}: \mathrm{B}=\mathrm{C}: \mathrm{D}$, s. oben) sind bei einer analogen Enkodierung sowohl Ziel- als auch Basisbereich aus dem Alltag nicht vertraut (vgl. Ferguson 1994; Gentner et al. 2009). Die Effektivität von analogen Enkodierungen konnte jedoch in verschiedenen Studien nachgewiesen werden (z. B. Gentner et al. 2003; Sagi et al. 2012). Allerdings gibt es dabei Unterschiede im Lerngewinn, die sich auf das Vorwissen der Lernenden zurückführen lassen: Novizen in einem Inhaltsgebiet legen ihren Schwerpunkt beim Mapping stärker auf Oberflächenmerkmale als auf 
die Tiefenstruktur (Chi et al. 1981). Enkodierungsprozesse sind jedoch um so erfolgreicher, je stärker die Analogien auf der Tiefenstrukturebene gemappt werden. Vosniadou (2001) und Posner et al. (1982) nehmen zwar lernförderliche Wirkungen von Analogiebildungen an; allerdings sind Analogiemodelle für Konzeptwechsel nur dann lernförderlich, wenn diese bestimmten Voraussetzungen genügen. Durch Vereinfachungen können Analogiemodelle sogar Lernprobleme verursachen (Kircher et al. 2009).

Der Lernerfolg bei Mapping-Prozessen durch Analogiebildung ist jedoch noch von weiteren Faktoren abhängig: Untersuchungen in der Grundschule zum Hebelgesetz zeigen, dass der Lerngewinn, der durch die Mapping-Prozesse in einem am analogen Enkodieren ausgerichteten Unterricht erreicht wird, stärker vom Vorwissen der Kinder als von ihrer Intelligenz abhängt (Lohrmann et al. 2018). Zudem ist das Mapping zwischen zwei Konkretionen v.a. auf das inhaltsspezifische Vorwissen zurückzuführen (Rittle-Johnson und Star 2009). Studien von Möller et al. (2002) deuten darauf hin, dass für leistungsschwache Schülerinnen und Schüler im Grundschulalter insbesondere strukturierende Maßnahmen besonders erfolgreich sind. Dies gilt auch für einen Unterricht, der an der Idee des analogen Enkodierens ausgerichtet ist, um eine Fokussierung auf wesentliche Elemente des Lerngegenstandes zu erreichen (vgl. Hardy 2012; Lohrmann et al. 2014). Strukturierende Maßnahmen sind vor allem zu Beginn eines Analogiebildungsprozesses erforderlich, aber auch bei der Spezifizierung der Lernziele, damit Analogieschlüsse korrekt gezogen werden können. Dies ist auch ein Anspruch an die in der vorliegenden Untersuchung eingesetzten Analogiemodelle.

\subsection{Analogiebildung beim Thema Stromkreis}

Beim Thema Stromkreis ergibt sich die Herausforderung, dass Schülerinnen und Schüler der Primarstufe in der Regel aus ihren Alltagserfahrungen bereits Vorstellungen bezüglich Stromfluss und Stromverbrauch aufgebaut haben. Diese sind allerdings zum Großteil aus fachwissenschaftlicher Sicht nicht belastbar. So ist bei der Mehrzahl der Kinder hinsichtlich des Stromflusses (im Stromkreis) eine Einwegzuführungsvorstellung bzw. eine Zweiwegezuführungsvorstellung feststellbar (vgl. u. a. Haider 2010; Wiesner 1995). Diese Kinder gehen zunächst davon aus, dass es eines einzigen Anschlusses bedarf, um ein Lämpchen zum Leuchten oder einen Elektromotor in Bewegung zu bringen. Erfahren sie in einem Experiment, dass dies nicht funktioniert, so vermuten sie - durch die Übertragung von Alltagserfahrungen -, dass eine zu geringe Strommenge den Verbraucher erreicht, was durch zwei Zuführungsdrähte gelöst werden kann (vgl. Haider 2010; Wiesner 1995). Mit beiden Vorstellungen zum Stromfluss geht eine Stromverbrauchsvorstellung einher: Der Strom wird im Lämpchen verbraucht - ähnlich wie Brennholz bei einem Lagerfeuer verbraucht wird. Zum Teil nehmen Kinder auch an, dass ausgehend von Plusund Minuspolen unterschiedliche Stoffe zum Lämpchen fließen und dort verbraucht werden (vgl. Haider 2010; Kircher 1989, 1995; Wiesner 1995).

Dass Strom im (Gleich-)Stromkreis zirkulär fließt, und dies auch noch sehr langsam, erscheint unplausibel. Hintergrund vieler dieser wissenschaftlich nicht korrekten Vorstellungen ist, dass Strom als etwas Substanzartiges und nicht als Prozess 
aufgefasst wird (vgl. z.B. Wiesner 1995). Schülerinnen und Schüler kennen das Problem, wenn im Alltag der „Nachschub“ fehlt und übertragen dies auf die Situation Lämpchen. Wenn die Substanz Strom fehlt, die ihrer Meinung nach zu Licht verwandelt wird, dann kann diese Verwandlung nicht stattfinden. Da die Bewegung der Elektronen nicht durch direkte Beobachtungen überprüft werden kann, ist der elektrische Stromkreis nur schwer nachzuvollziehen. Die mit der fehlenden Anschauung verbundene geringere Verständlichkeit ist einer der Hauptgründe, weswegen es schwierig ist, Vorstellungen von Schülerinnen und Schülern im Grundschulalter zu diesem Themenschwerpunkt zu verändern (vgl. u.a. Haider 2010; Kircher 1989; Wiesner 1995). Das Verständnis für einen zirkulären Stromfluss kann jedoch leichter erworben werden als korrekte Vorstellungen von Energieumwandlung, die ein Stromverbrauchskonzept ersetzen müssten (ebd.). Mehr noch: Ein korrektes Flusskonzept kann als Voraussetzung für das Verständnis von Energieumwandlung gesehen werden; erst durch das Verständnis eines zirkulären Stromflusses und dem damit verbundenen Wissen über die gleiche Stromstärke an jedem Punkt des Stromkreises wird deutlich, dass Strom nicht verbraucht wird. Das korrekte Energieumwandlungskonzept kann dann, sofern es als verständliche und plausible Alternative zum Stromverbrauch dargeboten wird, aufgebaut werden.

Um die Lernschwierigkeiten beim Thema Stromkreis zu überwinden, können unterschiedliche Analogiemodelle eingesetzt werden. Schon vor mehreren Jahrzehnten wurden diese in physikdidaktischen Veröffentlichungen gefordert - in erster Linie, um die fehlende Anschaulichkeit des Unterrichtsgegenstandes zu überwinden (vgl. u. a. Rhöneck und Völker 1982; Niedderer und Gohmert 1982). Für das Verständnis des einfachen Stromkreises werden Wassermodelle (mit einer Pumpe und Doppelwassersäulen), mechanische Modelle (Fahrradkettenmodell, Riemenmodell), Höhenanalogien (z. B. das Stäbchenmodell von Wilhelm, 2015) oder gespielte Analogien (Kircher et al. 2009) vorgeschlagen (vgl. zsf. Haider 2010, S. 68 f.). Diese Modelle haben spezifische Vor- und Nachteile, und sie unterscheiden sich mit Blick auf die Oberflächen- und die Tiefenstruktur.

Insbesondere mit Hilfe von Wassermodellen wurden bereits verschiedene Untersuchungen zum Verständnis des Stromkreises durchgeführt (vgl. Haider 2010). Bei Wassermodellen wird die Energiequelle mit Hilfe einer Doppelwassersäule oder einer Handpumpe dargestellt, der Energiewandler (Lämpchen oder Elektromotor) in Form eines Wasserrades. Der Elektronenfluss wird durch den Wasserfluss symbolisiert; die durch den unterschiedlichen Wasserstand erzeugte Druckdifferenz setzt das Wasserrad in Bewegung. Die Untersuchungen zu unterschiedlichen unterrichtlichen Zielen fanden überwiegend in der Sekundarstufe statt und lieferten unterschiedliche Ergebnisse hinsichtlich ihrer Effektivität hinsichtlich des Kompetenzerwerbs (vgl. u.a. Schwedes 1985; Dudeck 1997; zsf. Haider 2010). Sie lassen nur wenige begründbare Rückschlüsse auf Lernprozesse bei Kindern im Grundschulalter $\mathrm{zu}$, da diese in der Regel über ein geringeres Vorwissen verfügen. Zudem ist der Einsatz von Wassermodellen nicht unumstritten - kritisiert wird v.a., dass bei den Kindern die Vertrautheit mit geschlossenen Wasserkreisen fehle und dass sie zu wenig Verständnis für (Wasser-)Druck haben (Burde und Wilhelm 2016a). Jedoch unterstreichen Burde und Wilhelm (2016b) auch, dass das Modell erfolgreich sein kann: „In der physikdidaktischen Forschung hat sich gezeigt, dass insbesondere sol- 
che Unterrichtskonzepte vergleichsweise lernfördernd sind, die auf dem elektrischen Potenzial aufbauen und dieses mithilfe eines Modells anschaulich visualisieren (wie beispielsweise der geschlossene Wasserkreislauf mit Doppelwassersäule)“ (S. 18). Beim mechanischen Riemenmodell wird mit Hilfe einer Kurbel ein kleiner Propeller, der über einen umlaufenden Gummiriemen mit der Kurbel verbunden ist, in Bewegung gesetzt. Die Kurbel stellt die Energiequelle dar, der Propeller den Energiewandler und der Riemen die sich bewegenden Elektronen.

Diese beiden in der vorliegenden Studie kontrastierten Modelle des Wasserkreislaufs und des Riemenmodells sind in Tab. 1 dargestellt. Beide Modelle bilden zwar alle für die Grundschule relevanten Aspekte ab, allerdings sind sie sowohl auf der Oberflächenstrukturebene als auch auf tiefenstruktureller Ebene unterschiedlich nahe an der Zielvorstellung, so dass die von Ruppert (2017) genannten Phasen der Analogien von den Schülerinnen und Schülern auch unterschiedlich gut erkannt werden (Haider 2010). Daher ist anzunehmen, dass die verschiedenen Modelle unterschiedlich wirken: Auf den ersten Blick scheint die Oberflächenanalogie beim Wasserkreis leichter nachvollziehbar und anschaulicher zu sein, denn das fließende Wasser ist in Kreisform mit „Quelle“ und „Verbraucher“ deutlich zu erkennen. Es werden zudem der Stromfluss (Umlauf des Wassers in den Schläuchen) und die Spannung (unterschiedlicher Wasserstand) veranschaulicht. Es lassen sich an diesem Modell anschaulich Analogien zur Batterie herstellen: Beim Ausgleich der beiden Wassersäulen ist keine Energie mehr für den Antrieb des Wasserrades vorhanden; dies entspricht einer ,leeren“ Batterie. Die tiefenstrukturelle Analogie könnte aber bei diesem Modell weniger leicht nachvollziehbar sein. So enthält das Doppelwassersäulenmodell einen zusätzlichen Abstraktionsanspruch und ist komplexer, denn es muss die Differenz zweier potentieller Energien der Doppelwassersäule, die von den Schülerinnen und Schülern nicht aktiv erfahren wird, als Energiequelle erkannt werden. Die Oberflächenanalogie ist auch beim Riemenmodell gut nachvollziehbar; anders als beim Wassermodell ist hier allerdings das Fließen (Stromfluss) nicht direkt erkennbar. Dafür kann jedoch möglicherweise die strukturelle Analogie beim mechanischen Kreis leichter nachempfunden werden, da die Umwandlung von mechanischer Energie (kurbeln) in Bewegungsenergie (Propeller) auch haptisch erfahren wird. Dass der Riemen erhalten bleibt, ist zudem deutlicher erkennbar als die Erhaltung des Wassers. Des Weiteren ist der mechanische Kreis nicht durch Ausdrücke in der Alltagssprache (wie etwa Wasserverbrauch) beeinflusst, weswegen z.B. Muckenfuß (1995) dieses Modell vorzieht. Diese Präferenz lässt sich auch aus der Structure Mapping Theory (Gentner 1983) erklären, da Modelle, die die strukturellen Merkmale des Stromkreises nachvollziehbarer abbilden, beim Lernen komplexerer Lerngegenstände (z. B. beim Verbrauchskonzept) besser geeignet sein müssten als Modelle, bei denen diese weniger anschaulich erkennbar sind. Für einfachere Aspekte hingegen (wie z.B. die Veranschaulichung des Stromflusses) dürften beide Analogiemodelle geeignete Anschauungshilfen sein.

Bislang existiert lediglich eine Studie über den Einsatz von Analogiemodellen im Unterricht zum Stromkreis in der Grundschule (Haider 2010). Die Befunde deuten darauf hin, dass in der Summe durch die Kombination zweier unterschiedlicher Modellarten kein erhöhter Lernzuwachs bei den Schülerinnen und Schülern entstand. Allerdings gibt es bereits erste Hinweise darauf, dass Lernende unterschiedlicher 


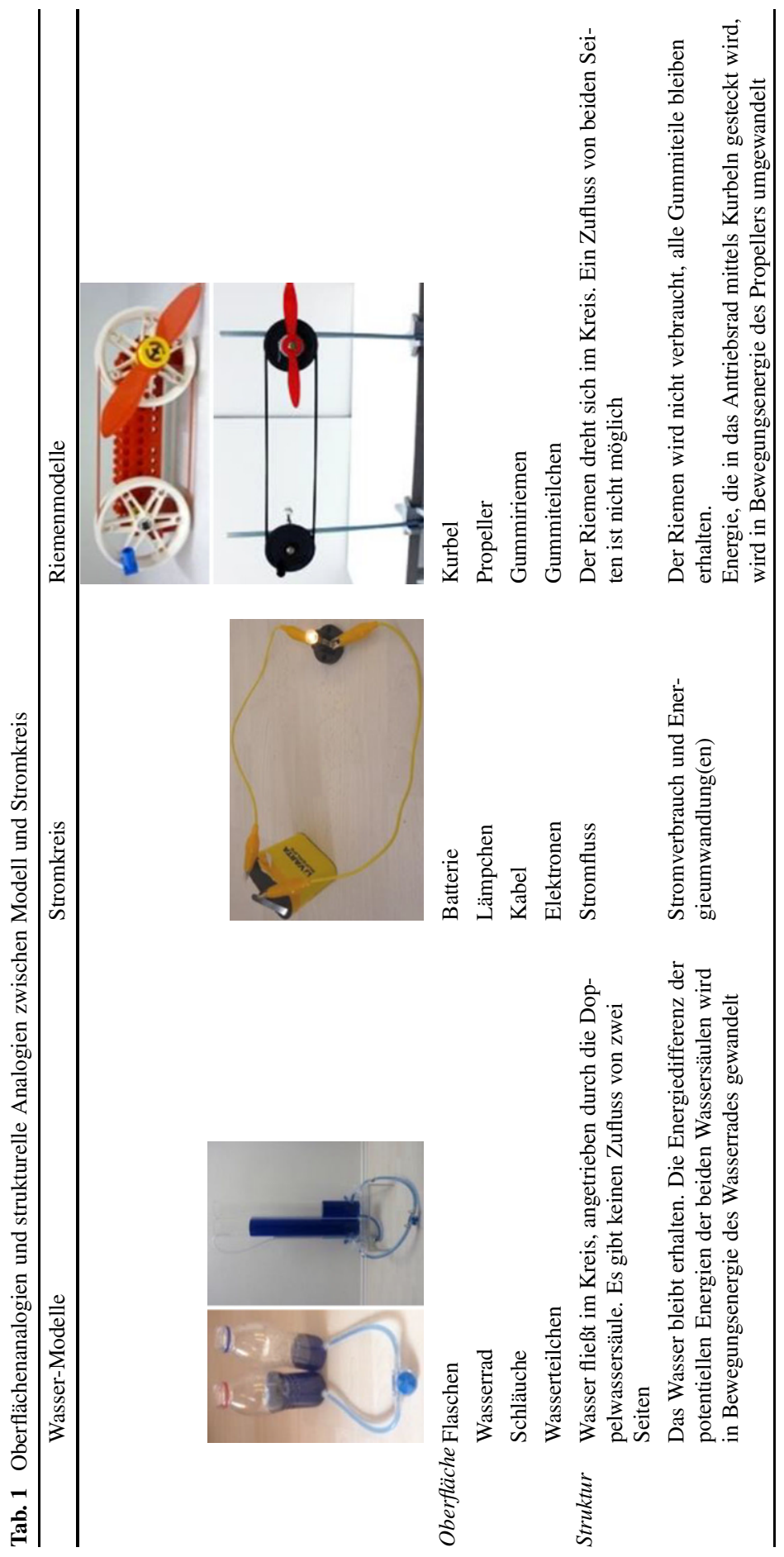


Leistungsgruppen vom Einsatz der Analogien unterschiedlich profitieren. Die Befunde bei Haider (2010) unterliegen allerdings der Limitation, dass der Unterricht von den jeweiligen Klassenlehrpersonen durchgeführt worden war. Diese hatten zuvor im Rahmen einer Fortbildung die Inhalte der Intervention kennen gelernt und erhielten für die einzelnen Lerneinheiten den konkreten Stundenablauf mit den erforderlichen Unterrichtsmedien sowie eine Anleitung zu deren Einsatz. Der vergleichsweisen hohen ökologischen Validität des Studiendesigns steht aber eine große Varianz des Unterrichtsgeschehens entgegen, da trotz der Vorgaben die konkrete Umsetzung einer individuellen Interpretation unterliegt. Die Zahl der Unterrichtsstunden war in den Versuchsgruppen und Kontrollgruppen zwar gleich, allerdings führten die Kontrollgruppen ihren Unterricht ohne spezielle Anweisungen und vorgegebene Unterrichtsmedien durch. In der vorliegenden Studie hingegen werden die Effekte des Lernens mit Analogiemodellen in einem Design unter Kontrolle der Varianz der Lehrperson untersucht.

\section{Fragestellungen und Hypothesen}

Es ist für Kinder im Grundschulalter sehr schwierig, belastbare Konzepte zum Thema elektrischer Strom aufzubauen, da diese eine anspruchsvolle theoretische Modellierung erfordern. Zudem müssen wissenschaftlich nicht anschlussfähige Vorstellungen (z.B. zum Stromfluss die Einweg- bzw. Zweiwegezuführungsvorstellung; zum Stromverbrauch die Verbrauchsvorstellung) abgebaut und es muss fachlich belastbares konzeptuelles Wissen zum Stromkreis aufgebaut werden. Ziel dieser Studie ist zu untersuchen, inwieweit durch den Einsatz unterschiedlicher Analogiemodelle bei verschiedenen Schülergruppen ein solcher Zuwachs im konzeptuellen Wissen unterstützt wird. Folgende Fragen werden untersucht:

1. Führt die Verwendung von Analogiemodellen im Unterricht zum Thema Strom zu einem stärkeren Zuwachs an konzeptuellem Wissen bei Schülerinnen und Schülern der Grundschule als Unterricht ohne Analogiemodelle?

H1) Da Analogiemodelle die Möglichkeit zur Veranschaulichung bieten, wird erwartet, dass der Einsatz von Analogiemodellen dazu führt, dass von den Schülerinnen und Schülern konzeptuelles Wissen auf einem höheren Niveau aufgebaut wird als von den Schülerinnen und Schülern, die ohne Analogiemodelle unterrichtet werden.

H2) Ein höherer Zuwachs im konzeptuellen Wissen wird insbesondere für das Teilthema Stromverbrauch erwartet, da hier die Anschauung von besonderer Bedeutung ist, um dem - auch durch die Alltagssprache verursachten - Fehlkonzept entgegen zu treten.

2. Führen die Analogiemodelle Wassermodell versus Riemenmodell mit ihren unterschiedlichen Oberflächen- und Tiefenstrukturmerkmalen zu einem unterschiedlichen Zuwachs des konzeptuellen Wissens? 
H3) Mit Blick auf das leichter verständliche Teilthema Stromfluss werden keine Unterschiede erwartet, da beide Analogiemodelle eine vergleichbar anschauliche Oberflächenstruktur haben und mit Blick auf die Tiefenstruktur das Fließen des Stroms gleichermaßen repräsentieren können.

H4) Beim komplexeren Teilthema Stromverbrauch wird ein höherer Lernzuwachs beim Einsatz des Riemenmodells erwartet, da hier der Fokus auf strukturellen Merkmalen liegt und dies gemäß der Structure Mapping Theory zu besseren Ergebnissen führt.

3. Inwieweit profitieren Kinder mit ungünstigen Lernvoraussetzungen unterschiedlich von den Analogiemodellen?

H5) Aufgrund ihrer Anschaulichkeit sollten Analogiemodelle insbesondere Kindern mit ungünstigen Lernvoraussetzungen helfen, anschlussfähiges konzeptuelles Wissen aufzubauen. Daher gehen wir davon aus, dass diese Kinder im Unterschied zur Vergleichsgruppe ohne Analogiemodelle in besonderer Weise profitieren.

\subsection{Anlage der Untersuchung}

Um den Einfluss unterschiedlicher Analogiemodelle auf den Zuwachs im konzeptuellen Wissen der Schülerinnen und Schüler zu den Schwerpunkten Stromfluss und Stromverbrauch zu untersuchen, wurde mit einem quasi-experimentellen Design eine Unterrichtsreihe durchgeführt. In zwei Versuchsgruppen wurde jeweils ein bestimmtes Analogiemodell verwendet (VS1, Wassermodell; VS2, Riemenmodell), in der Vergleichsgruppe (VG) fand der Unterricht ohne den Einsatz von Modellen statt. Eine Wartekontrollgruppe (WG) sicherte den Einfluss der Messinstrumente ab (Abb. 1).

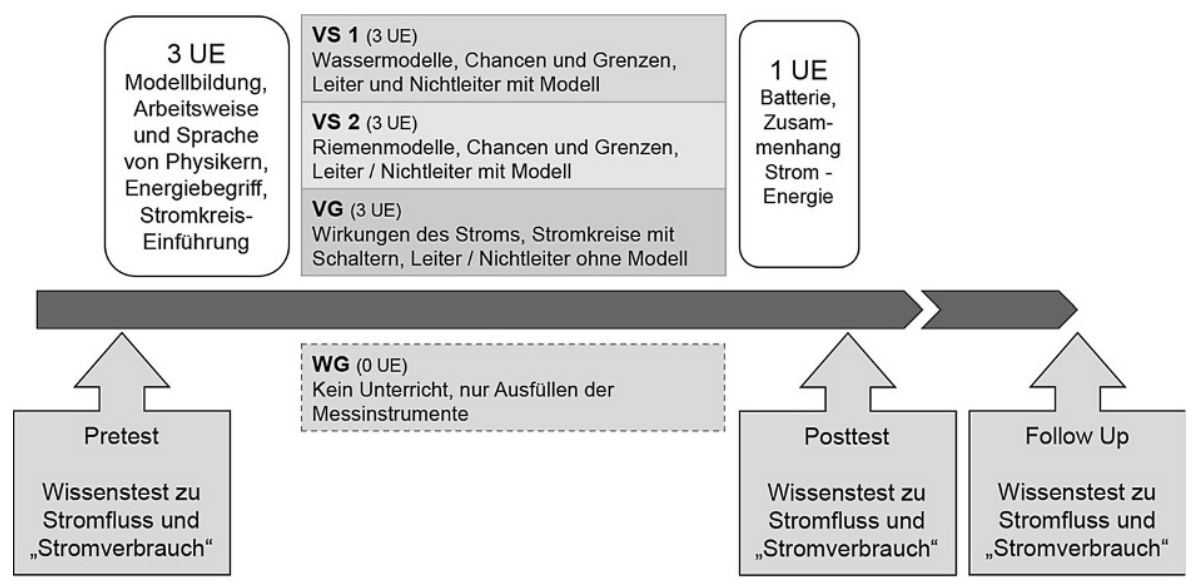

Abb. 1 Design der Studie 
Zur Erfassung des konzeptuellen Wissens wurden Fragebögen für Schülerinnen und Schüler der dritten Jahrgangsstufe konstruiert. Der quantitative Zugang wurde gewählt, um die Entwicklung in der Gesamtgruppe, in den Experimental- und Vergleichsgruppen sowie in den genannten Subgruppen aufzuzeigen. Hierzu lagen aus Vorstudien (z. B. Haider 2010) Ergebnisse vor, sodass hypothesentestend vorgegangen werden konnte. Die Follow-up-Erhebung erfolgte sechs Monate nach Beendigung der Intervention.

\subsection{Stichprobe}

Zur Untersuchung der Hypothesen wurden aus zunächst 20 Klassen über Vortests 16 Klassen mit vergleichbaren Voraussetzungen bzgl. des Vorwissens zu Stromfluss und Stromverbrauch ausgewählt und zufällig den drei Untersuchungsgruppen und einer Wartegruppe zugeordnet. Die Größe der Stichproben waren mit $N_{\mathrm{Vs} 1}=86$, $N_{\mathrm{VS} 2}=81, N_{\mathrm{VG}}=87, N_{\mathrm{WG}}=87$ ungefähr gleich groß. Das mittlere Alter lag bei $M=8,4$, $S D=0,54\left(M_{V S 1}=8,4, S D_{V S 1}=0,52 ; M_{V S 2}=8,4, S D_{V S 2}=0,54 ; M_{V G}=8,44, S D_{V G}=0,57\right.$; $\left.M_{W G}=8,37, S D_{W G}=0,57\right)$. Es wurde auf eine Ausgewogenheit in der Verteilung von Mädchen und Jungen (VS1: 45 Mädchen, 41 Jungen; VS2: 37 Mädchen, 44 Jungen; VG: 48 Mädchen, 39 Jungen; WG: 40 Mädchen, 47 Jungen) geachtet.

\subsection{Intervention}

Die Intervention umfasste 7 Unterrichtsteileinheiten mit in der Regel 90 min Unterrichtszeit. Der Unterricht wurde in allen Interventionsgruppen von einer geschulten Grundschullehrerin mit hoher Expertise im Bereich des naturwissenschaftlichen Sachunterrichts durchgeführt. Damit konnte die Variation zwischen den Bedingungen in Bezug auf die Erfahrungen der Schülerinnen und Schüler mit ihrer Klassenlehrkraft ausgeschlossen werden. Die Unterrichtseinheiten wurden im Vorfeld so geplant und konzipiert, dass sie sich ausschließlich bzgl. der Analogiemodelle unterschieden, mit Blick auf Inhalte, zeitlichen Ablauf und Methoden jedoch identisch waren. Um zu überprüfen, inwieweit diese Unterrichtsplanung entsprechend umgesetzt wurde, wurden sämtliche Unterrichtseinheiten videographiert und von geschulten Mitarbeitern und Mitarbeiterinnen hinsichtlich der Umsetzung der Inhalte analysiert. Diese Analyse ergab, dass diese Umsetzung in den Bedingungen VS1, VS2 und VG gelungen ist.

Die ersten drei Unterrichtseinheiten behandelten in allen Versuchsgruppen (VS1, VS2, VG) die gleichen Themen. Die Schülerinnen und Schüler erhielten erste Einblicke in naturwissenschaftliche Arbeitsweisen und erfuhren Modelle als Möglichkeit zur Erklärung von Versuchsergebnissen. Durch das Kennenlernen beispielhafter Modelle und eine Verknüpfung zum Vorwissen wurden der Modellbegriff, Arten von Modellen, Unterschiede zwischen Modell und Original, Nutzen von Modellen als Erklärungs-, Veranschaulichungs- und Verständnishilfe sowie deren Grenzen erarbeitet. Um durch eine angemessene Energievorstellung in den Folgestunden ein anschlussfähiges Verständnis des Stromkreises zu unterstützen, wurden verschiedene Aspekte zu Energie erarbeitet. Die Schülerinnen und Schüler lernten Wirkungen der Energie, verschiedene Energieformen und Energieumwandlungsprozesse ken- 
nen. In der dritten Unterrichtseinheit lag der Fokus auf dem Aufbau eines einfachen Stromkreises.

In den folgenden drei Einheiten bestand die Variation darin, ob bzw. welche Analogiemodelle eingesetzt wurden. Die Versuchsgruppen VS1 und VS2 arbeiteten entweder mit Wasser- oder mit Riemenmodellen (vgl. Tab. 1), erfuhren Möglichkeiten und Grenzen von Modellen als Veranschaulichungshilfe und erarbeiteten sich (mit Hilfe von Modellen) eine Vorstellung von Leitern und Nichtleitern.

Das Wassermodell (VS1) wurde in zwei Varianten eingesetzt: Die Schülerinnen und Schüler arbeiteten mit einem Modell, bei dem an zwei Flaschen mit unterschiedlichem Wasserstand Schläuche befestigt wurden, die wiederum mit einem Wasserrad verbunden waren. Zusätzlich gab es ein Doppelwassersäulenmodell als großes Anschauungsmodell. Durch beide Modelle sollte verdeutlicht werden, dass die durch den unterschiedlichen Wasserstand erzeugte Druckdifferenz das Wasserrad in Bewegung setzt.

Beim Riemenmodell (VS2) wurde mit Hilfe einer Kurbel ein kleiner Propeller, der über einen umlaufenden Gummiriemen mit der Kurbel verbunden war, in Bewegung gesetzt. Auch dieses Modell wurde in der Untersuchung in zwei Ausführungen eingesetzt - zunächst mit Hilfe von Legosteinen für die eigenaktive Auseinandersetzung, im späteren Unterrichtsverlauf als großes Anschauungsmodell für die gesamte Klasse zur Wiederholung und Vertiefung.

Beide Modelle dienten dem Aufbau eines konzeptuellen Verständnisses, da bei der Betrachtung der Modelle vorstellbar wird, dass Strom analog zum rundum fließenden Wasser bzw. Gummiriemen im Kreis fließt und dabei kein Verbrauch stattfindet, sondern Energieumwandlungsprozesse (initiiert durch den Höhenunterschied der Wassersäulen bzw. das Kurbeln) ablaufen. Durch den Einsatz dieser beiden Modelltypen sollten wesentliche, für den Stromkreis grundlegende Elemente auf der Oberflächenebene und auf der strukturellen Ebene (s.Tab. 1) veranschaulicht werden.

Beim Riemenmodell kann gezeigt werden, dass sich der Riemen im Kreis dreht und keine auf dem Riemen aufgezeichneten Elektronen verloren gehen (verbraucht werden). Im (Doppelwassersäulen-)Wassermodell kann nachvollzogen werden, dass das Wasser, das ein Wasserrad antreibt, in den Schläuchen so lange im Kreis fließt, bis der Wasserstand in den Wassersäulen ausgeglichen ist; es wird kein Wasser verbraucht.

Bei beiden Analogiemodellen kann somit auf Ähnlichkeitsmerkmale (Oberfläche) und auf strukturelle Merkmale (z.B. Elektronenfluss, Energieübertragung) fokussiert werden; es können mit beiden Modellen die Strukturmerkmale Stromfluss und Stromverbrauch nachvollziehbar veranschaulicht werden. Die Inhalte, auf die die Aufmerksamkeit zu richten ist, sind dabei: Energieübertragung, Bewegung von Elektronen und „Strom als Prozess“.

Die Vergleichsgruppe (VG) erhielt einen Unterricht über Wirkungen des Stroms, Stromkreise mit Schaltern sowie Leiter und Nichtleiter ohne Modelle und Analogiemodelle. In der UE 4 wurden Versuche zu unterschiedlichen Wirkungen des Stroms durchgeführt. In der UE 5 wurde mit Hilfe verschiedener Versuche erarbeitet, dass ein Schalter den Stromkreis unterbricht (offener, geschlossener Stromkreis). Mit Hilfe verschiedener Materialien wurde erprobt, welche Stoffe als Schalter geeignet 
Tab. 2 Unterrichtssequenz zum Thema Strom

\begin{tabular}{llll}
\hline & VS 1 (Wassermodell) & VS 2 (Riemenmodell) & VG (ohne Modell) \\
\hline UE 1 & Arbeitsweise von Physikern; Einführung Modellbegriff & \\
UE 2 & Einführung „Energie“ Sprache von Physikern & \\
UE 3 & Bau eines einfachen Stromkreises & & \\
UE 4 & Wassermodell & Riemenmodell & \\
& für den Stromkreis & für den Stromkreis & Wirkungen des \\
UE 5 & Vorteile und Grenzen & Vorteile und Grenzen & Stroms \\
& des Wassermodells & des Riemenmodells & Stromkreise mit \\
UE 6 & $\begin{array}{l}\text { Leiter und Nichtleiter } \\
\text { (modellgestützt) }\end{array}$ & $\begin{array}{l}\text { Leiter und Nichtleiter } \\
\text { (modellgestützt) }\end{array}$ & Schaltern \\
UE 7 & Batterie; Zusammenhang von Strom und Energie & Leiter und Nichtleiter \\
\hline
\end{tabular}

sind und wie Schalter im Stromkreis funktionieren. Es wurde nochmals thematisiert, dass man von Strom spricht, wenn Elektronen im Kabel fließen, dass diese im Kreis fließen und nicht verbraucht werden. Somit wurde sichergestellt, dass in der Vergleichsgruppe alle Inhalte vergleichbar zu den Versuchsgruppen vermittelt wurden. In der UE 6 ging es in allen Gruppen (VS1, VS2, VG) um die Frage, welche Stoffe Strom leiten. In einer Diskussion der Gruppenarbeiten erfuhren die Schülerinnen und Schüler der VG, dass z. B. Metalle Stoffe sind, in denen sich Elektronen bewegen können und die Atome anstoßen und diese deshalb Strom leiten (Leiter).

Den Abschluss der Intervention bildete eine in allen Gruppen gleiche Unterrichtseinheit mit Fokus auf der Unterscheidung von Strom und Energie und der Bearbeitung der Frage, warum eine Batterie „leer“ wird, wenn Strom im Kreis fließt und nicht verbraucht wird (Energieumwandlungskonzept). Abschließend sollte der Abbau des durch die Alltagssprache implizierten Stromverbrauchskonzepts noch einmal durch die Reflexion und Gegenüberstellung von wissenschaftlich anders verwendeten Alltagsbegriffen (Stromrechnung, Strom sparen) und physikalischer Fachsprache unterstützt werden. In allen Gruppen wurde ein handlungsintensiver Unterricht durchgeführt, in dem die Vorkenntnisse und Vorerfahrungen der Schülerinnen und Schüler berücksichtigt und auf ihre Fragen differenziert eingegangen wurde. Die Wartekontrollgruppe (WG) erhielt zwischen Prä- und Posttest keinen Unterricht zum Stromkreis und diente der Prüfung der Effekte des Messinstruments. Diese Schülerinnen und Schüler erhielten nach dem Posttest die Intervention und standen somit für die Follow-up-Untersuchung nicht mehr zur Verfügung (tabellarische Aufstellung siehe Tab. 2).

\subsection{Messinstrument: Fragebogen zur Erfassung des konzeptuellen Wissens zum Thema Strom}

Mit allen Schülerinnen und Schülern wurden zu allen Messzeitpunkten (Ausnahme: WG am MZP 3) identische, sich wiederholende schriftliche Befragungen zum konzeptuellen Wissen durchgeführt. Der Fragebogen wurde in wesentlichen Teilen der Studie von Haider (2010) entnommen, allerdings erweitert und in Vorstudien überprüft. Es wurden die beiden Subskalen Stromfluss und Stromverbrauch mit je 4 Items gebildet. Um die interne Konsistenz zu bestimmen, wurde Cronbachs Alpha 
Tab. 3 Deskriptive Werte des Tests zum konzeptuellen Wissen

\begin{tabular}{lllllllll}
\hline Skala & MZP & $n$ & Anzahl Items & Min & Max & $M W$ & $S D$ & $\alpha$ \\
\hline Stromfluss- & Prätest & 341 & 4 & 0 & 2 & 0,88 & 0,46 & 0,54 \\
vorstellun- & Posttest & 341 & 4 & 0 & 2 & 1,64 & 0,52 & 0,83 \\
gen & Follow-up & 254 & 4 & 0 & 2 & 1,77 & 0,40 & 0,76 \\
\multirow{2}{*}{ Stromver- } & Prätest & 341 & 4 & 0 & 2 & 1,00 & 0,72 & 0,82 \\
brauchs- & Posttest & 341 & 4 & 0 & 2 & 1,60 & 0,60 & 0,86 \\
vorstellungen & Follow-up & 254 & 4 & 0 & 2 & 1,52 & 0,64 & 0,85 \\
\hline
\end{tabular}

für die Subskalen Stromfluss und Stromverbrauch berechnet. Die interne Konsistenz der Skala war (mit Ausnahme des Prätests bzgl. der Stromflussvorstellungen) mit Werten von Cronbachs Alpha von etwa 0,80 hoch. Lediglich der Prätestwert zur Skala Stromflussvorstellungen ist als nicht zufriedenstellend einzustufen (siehe Tab. 3).

Die Fragen wurden zu 75\% als Multiple-Choice-Aufgaben formuliert. Die einzelnen Items zum Stromfluss und zum Stromverbrauch wurden an unterschiedlichen Anwendungssituationen (z. B. Lämpchen, Spielzeughubschrauber) festgemacht und zielten auf die Unterscheidung einzelner aus der Theorie bekannter Vorstellungen wie Einwegzuführung vs. Zweiwegezuführung vs. Kreisfluss bzw. Stromverbrauch vs. Energieumwandlung vs. Elektronenverbrauch. Diese sollten in den verschiedenen Situationen gegeneinander abgewogen werden.

Für die Skala Stromfluss musste z.B. zweimal eine bildliche Darstellung ergänzt werden: „Verbinde die Batterie so mit dem Lämpchen, dass es leuchtet! Zeichne in das Bild Pfeile ein, in welcher Richtung der Strom fließt.“. Ein Beispiel für eines der beiden Textitems ist: „Wie bringt man ein Lämpchen zum Leuchten? Wer hat recht? Max meint: Ich brauche ein Kabel, um ein Lämpchen mit der Batterie zu verbinden; Lena meint: Ich brauche zwei Kabel, weil dann durch beide Drähte Strom zum Lämpchen kommt; Susi meint: Ich brauche zwei Drähte, weil Strom aus der Batterie zum Lämpchen und wieder zur Batterie zurückfließt.“ Für die Skala Stromverbrauch wurden verschiedene Situationen, z. B. ein leuchtendes Lämpchen, die Rechnung des E-Werks, stehenbleibende Rotorblätter eines Hubschraubers oder Erklären einer „leeren“ Batterie verwandt. Ein Beispielitem für die Skala Stromverbrauch lautet „Der Propeller an Vincents Hubschrauber wird immer langsamer und bleibt schließlich stehen. Kreuze die passendste Erklärung an! Der Motor hat A) die Elektronen aus der Batterie verbraucht. B) den Strom aus der Batterie verbraucht. C) die Energie aus der Batterie verbraucht." Fehlende Messwerte wurden nach Kontrolle der Fehlerquote $(<10 \%)$ mit Hilfe des E/M-Algorithmus aus SPSS geschätzt.

Zur Prüfung der aus der Theorie abgeleiteten zweifaktoriellen Struktur wurde mit den Werten des Posttests eine konfirmatorische Faktorenanalyse mit der Gesamtstichprobe von $N=341$ gerechnet. Als Schätzmethode diente die Maximum-Likelihood-Methode. Der Modellfit wurde anhand der Ergebnisse des chi $^{2}$ Tests $\left(\chi^{2}=32,11, d f=17, p<0,05\right)$ und ausgewählter Fit-Indizes $(R M S E A=0,057$, $C F I=0,986)$ beurteilt. Die Konstrukte Stromfluss und Stromverbrauch wurden dabei latent modelliert. Die Faktorenladungen der Items auf den latenten Faktoren liegen 


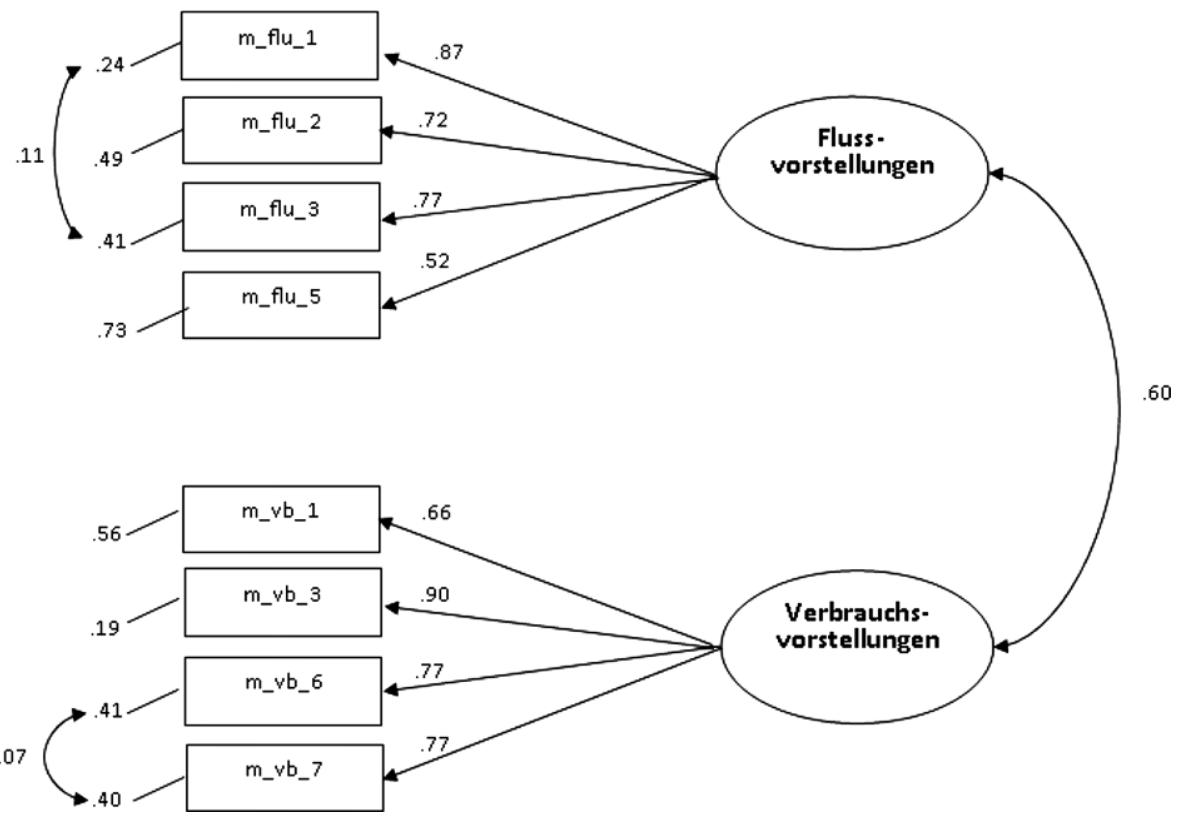

Abb. 2 Ergebnisse der CFA für zweifaktorielles Modell des Posttests zum konzeptuellen Wissen

zwischen 0,52 und 0,90 (s. Abb. 2). Dieses theoretisch abgeleitete zweifaktorielle Modell bildet die Datenstruktur deutlich besser ab als ein einfaktorielles Modell $\left(\chi^{2}=335,63, d f=20, p<0,001, R M S E A=0,242, C F I=0,711\right)$.

Zur Einschätzung der Schulleistung wurden Noten aus den letzten Zeugnisdaten erfasst. Gerechnet wurde mit der Durchschnittsnote aus den Fächern Deutsch, Mathematik und Heimat- und Sachunterricht.

\section{Ergebnisse}

\subsection{Effekte des Modelleinsatzes}

Zur Prüfung der Effekte des Modelleinsatzes (H1 und H2) wurde eine multivariate Varianzanalyse mit Messwiederholung und geplanten Kontrasten durchgeführt. Als abhängige Variablen gingen die Ergebnisse der Skalen Stromfluss und Stromverbrauch im Prätest und Posttest in das Modell ein. Zur Kontrastierung wurden zum einen zur Überprüfung des Effekts der Messinstrumente die Wartegruppe gegen alle anderen Gruppen (als Untersuchungsgruppen zusammen genommene Gruppen VS1, VS2 und VG) getestet. Als Kontrast wurde in SPSS die einfache Kontrastierung gewählt. Zum anderen wurden die Versuchsgruppen (VS1 und VS2) gemeinsam gegen die Vergleichsgruppe (VG) getestet, um die Effekte der Modelle zu überprüfen. Als Messwiederholungsfaktor gingen der Messzeitpunkt vor und nach der Intervention ein. 
Tab. 4 Deskriptive Werte der Subskalen Stromfluss- und Stromverbrauchskonzept zu drei Messzeitpunkten

\begin{tabular}{llllll}
\hline & & $N$ & $\begin{array}{l}\text { Prätest } \\
\text { M (SD) }\end{array}$ & $\begin{array}{l}\text { Posttest } \\
\text { M (SD) }\end{array}$ & $\begin{array}{l}\text { Follow-up } \\
\text { M (SD) }\end{array}$ \\
\hline $\begin{array}{lllll}\text { Strom- } \\
\text { fluss }\end{array}$ & VS1 & 86 & $0,88(0,57)$ & $1,82(0,27)$ & $1,75(0,44)$ \\
& VS2 & 81 & $0,86(0,43)$ & $1,86(0,27)$ & $1,79(0,37)$ \\
& VG & 87 & $0,94(0,44)$ & $1,80(0,30)$ & $1,75(0,40)$ \\
Strom- & WG & 87 & $0,89(0,47)$ & $1,08(0,64)$ & - \\
verbrauch & VS1 & 86 & $1,05(0,76)$ & $1,80(0,43)$ & $1,40(0,67)$ \\
& VS2 & 81 & $0,97(0,74)$ & $1,87(0,31)$ & $1,64(0,58)$ \\
& VG & 87 & $0,96(0,74)$ & $1,78(0,46)$ & $1,53(0,63)$ \\
& WG & 87 & $1,04(0,73)$ & $1,00(0,67)$ & - \\
\hline
\end{tabular}

In der MANOVA zeigt sich ein signifikanter Anstieg der korrekten Vorstellungen zum Stromfluss und Stromverbrauch (Tab. 4) im Vergleich der ersten beiden Messzeitpunkte, $F(2,337)=251,77, p<0,001, \eta^{2}=0,65$.

Dieser Anstieg ist auf die unterrichteten Gruppen zurückzuführen: Die Tests der Innersubjektkontraste ergeben für den Interaktionseffekt Zeit X Gruppe signifikante Unterschiede im Anstieg korrekter Vorstellungen zwischen der Wartegruppe (WG) und den Untersuchungsgruppen (VS1, VS2, VG) bezüglich den Stromflussvorstellungen, $F(2,338)=60,44, p<0,001, \eta^{2}=0,26$ und den Stromverbrauchsvorstellungen, $F(2,338)=29,51, p<0,001, \eta^{2}=0,15$.

Um allgemein zu testen, inwieweit die Nutzung eines Modells einen Einfluss auf den Lerngewinn hat, wurden die beiden Gruppen, die mit Modellen gearbeitet haben (VS1 und VS2) mit der Vergleichsgruppe (VG) kontrastiert. Die Tests der Innersubjektkontraste ergeben weder beim Flusskonzept, $F(1,338)=2,18, p>0,05$, $\eta^{2}=0,00$ noch beim Verbrauchskonzept, $F(1,338)=0,03, p>0,05, \eta^{2}=0,00$ signifikante Effekte. Dies widerspricht dem in Hypothese 1 und 2 angenommenen Vorteil der Versuchsgruppen VS1 und VS2.

\subsection{Effekte unterschiedlicher Analogiemodelle}

Die Effekte der verschiedenen Analogiemodelle wurden ebenfalls mittels einer multivariaten Varianzanalyse mit Messwiederholung und geplanten Kontrasten überprüft. Als abhängige Variablen gingen die Ergebnisse der Skalen Stromfluss und Stromverbrauch in das Modell ein. Zur Kontrastierung wurden die beiden Versuchsgruppen VS1 und VS2 gegenübergestellt. Für die Analyse wurden nur jene 167 Fälle ausgewählt, die diesen beiden Gruppen angehören. Als Messwiederholungsfaktor gingen die Messzeitpunkte vor und nach der Intervention sowie der Followup-Test ein.

Das Gesamtmodell zeigt die signifikante Veränderung der korrekten Vorstellungen zu den Themen Stromfluss und Stromverbrauch zwischen den drei Messzeitpunkten, $F(4,162)=193,76, p<0,001, \eta^{2}=0,83$. Die Tests der Innersubjektkontraste des Interaktionseffektes Zeit X Gruppe ergeben bezüglich der Stromflussvorstellungen keine signifikanten Unterschiede für den Vergleich von Prätest und Post- 
test, $F(1,165)=0,56, p>0,05, \eta^{2}=0,00$, sowie von Prätest und Follow-up Test, $F(1,165)=0,53, p>0,05, \eta^{2}=0,00$. Dieses Ergebnis entspricht der Hypothese 3 .

Beim Stromverbrauch ergeben sich keine Unterschiede zwischen den Versuchsgruppen in der Kontrastierung der Messzeitpunkte von Prätest und Posttest, $F(1,165)=1,35, p>0,05, \eta^{2}=0,01$. Jedoch ergibt sich ein signifikanter Interaktionseffekt von Zeit X Gruppe im Vergleich des Follow-up-Tests mit dem Prätest, $F(1,165)=4,93, p<0,05, \eta^{2}=0,03$. Damit wird Hypothese 4 bestätigt. Aus den deskriptiven Daten lässt sich ablesen, dass die Gruppe, die mit dem mechanischen Modell gearbeitet hat, im Follow-up die besseren Werte erzielt.

\subsection{Effekte bei Kindern mit ungünstigen Lernvoraussetzungen}

Um zu überprüfen, inwieweit es spezifische Effekte für Schülerinnen und Schüler mit ungünstigen Lernvoraussetzungen gibt, wurden bei den abhängigen Variablen zum Vorwissen Stromfluss und Stromverbrauch sowie zur Durchschnittsnote Terzile gebildet und jeweils die Daten der Personen mit den ungünstigen Voraussetzungen, also des untersten Terzils, zur Berechnung verwendet, mit $N$ (Durchschnittsnote $)=65$, $N($ Stromfluss $)=88, N($ Stromverbrauch $)=86$. Um Alphafehlerkumulation zu vermeiden, wurde jeweils mit dem Gesamtmodell MANOVA mit abhängigen Variablen Stromfluss und Stromverbrauch, Kontrastierung von Versuchsgruppen und Vergleichsgruppe gerechnet. Die Analysen wurden für die unterschiedlichen Personengruppen (unteres Terzil Vorwissen Stromfluss, unteres Terzil Vorwissen Stromverbrauch und unteres Terzil Durchschnittsnote) separat ausgeführt.

Die Schülerinnen und Schüler mit den schlechtesten Durchschnittsnoten zeigen eine signifikante Veränderung der korrekten Vorstellungen zu den Themen Stromfluss und Stromverbrauch im Laufe der Messzeitpunkte mit einem signifikanten Effekt des Faktors Zeit, $F(4,59)=62,30, p<0,001, \eta^{2}=0,81$. Zwischen den Gruppen ergeben die Innersubjektkontraste für Zeit X Gruppe keinen signifikanten Effekt bezüglich der Veränderung der Stromflussvorstellungen von Prätest zu Posttest, $F(2,62)=0,75, p>0,05, \eta^{2}=0,02$ und von Prätest zu Follow Up, $F(2,62)=0,96, p>0,05, \eta^{2}=0,03$. Auch bei den Stromverbrauchsvorstellungen ergeben die Innersubjektkontraste für Zeit X Gruppe keine signifikanten Effekte von Prätest zu Posttest, $F(2,62)=0,60, p>0,05, \eta^{2}=0,02$ sowie von Prätest zu Follow Up, $F(2,62)=0,66, p>0,05, \eta^{2}=0,02$.

Bei der Subgruppe mit wenig Vorwissen zum Stromfluss und der Subgruppe mit wenig Vorwissen zum Stromverbrauch ergeben sich in der Aussage identische Befunde bezüglich der hier dargestellten Ergebnisse. Zusammenfassend ergibt die

Tab. 5 Übersicht zur Bestätigung der bzw. Abweichung von den aufgestellten Hypothesen

\begin{tabular}{|c|c|c|c|c|c|c|c|}
\hline \multicolumn{2}{|c|}{$\begin{array}{l}\text { Einsatz von Analogie- } \\
\text { modellen } \\
\text { Vergleich VS-VG }\end{array}$} & \multicolumn{2}{|c|}{$\begin{array}{l}\text { Vergleich zweier } \\
\text { Modelle } \\
\text { VS1-VS2 }\end{array}$} & \multicolumn{4}{|c|}{$\begin{array}{l}\text { Profitieren der Kinder mit ungünstigen Lernvoraus- } \\
\text { setzungen }\end{array}$} \\
\hline$H 1$ & $H 2$ & $H 3$ & H4 & H5 & & & \\
\hline Fluss & Verbrauch & Fluss & Verbrauch & Gerin & wissen & Niedri & ulleistung \\
\hline$x$ & $x$ & $\checkmark$ & $\checkmark$ & $\begin{array}{l}\text { Fluss } \\
\times\end{array}$ & $\begin{array}{l}\text { Verbrauch } \\
\times\end{array}$ & $\begin{array}{l}\text { Fluss } \\
\times\end{array}$ & $\begin{array}{l}\text { Verbrauch } \\
\times\end{array}$ \\
\hline
\end{tabular}


Einschränkung auf die Schülerinnen und Schüler mit ungünstigen Lernvoraussetzungen also keine signifikanten Unterschiede hinsichtlich des Lernerfolgs im Unterricht mit und ohne Modelle.

\section{Zusammenfassung und Diskussion}

Im Rahmen des Forschungsvorhabens sollte untersucht werden, ob bzw. inwieweit durch den Einsatz von Analogiemodellen der Aufbau eines Stromkreiskonzepts und der Abbau von Stromverbrauchsvorstellungen besser unterstützt werden kann als ohne den Einsatz entsprechender Modelle (vgl. Hypothesen 1 und 2). Dabei sollten die Effekte des Einsatzes von zwei unterschiedlichen Analogiemodellen (Wassermodelle vs. Riemenmodelle) verglichen werden (Hypothesen 3 und 4) und Auswirkungen auf den Aufbau konzeptuellen Wissens bei Schülerinnen und Schülern mit ungünstigen Lernvoraussetzungen (Vorwissen und Schulleistungen) untersucht werden (Hypothese 5). Dabei zeigte sich, dass nur manche Hypothesen theoriekonform bestätigt werden konnten (vgl. Tab. 5).

Insbesondere die nicht bestätigten Hypothesen sind aus unserer Sicht diskussionswürdig. Im Rahmen der Hypothesen 1 und 2 wurde davon ausgegangen, dass sich die Arbeit mit Modellen positiver auf die Lernergebnisse zum Stromfluss und -verbrauch auswirkt als ein Unterricht ohne den Einsatz von Analogiemodellen. Dies wurde durch die Untersuchung nicht bestätigt. Der sehr große Lerngewinn der Schülerinnen und Schüler, die ohne Analogiemodelle lernten, im konzeptuellen Subtest zum Stromfluss bedarf einer Erklärung - denn er steht im Gegensatz zu den Ergebnissen der Studie von Haider (2010), in der diese Gruppe deutlich geringere Lernerfolge erzielte als die Gruppe der mit Modellen lernenden Schülerinnen und Schüler. Gründe für den großen Lernerfolg der Vergleichsgruppe könnten auf die Art des hier durchgeführten Unterrichts zurückzuführen sein (vgl. dazu auch die Befunde und die Erklärungen bei Lohrmann et al. 2014). In allen Gruppen erhielten die Schülerinnen und Schüler einen handlungsintensiven Unterricht mit Versuchen in Kleingruppen. Die Arbeitsmaterialien waren vorab in anderen Schulklassen hinsichtlich ihrer Einsetzbarkeit erprobt worden. Zudem wurde der Unterricht in allen Gruppen von einer fachlich und fachdidaktisch versierten Lehrkraft ${ }^{1}$ durchgeführt. In der Untersuchung von Haider (2010) führten die Lehrerinnen und Lehrer der Vergleichsgruppe dagegen ihren üblichen Unterricht zum Thema Strom selbst durch. Ein fachlich und fachdidaktisch hochwertiger Unterricht, so scheint es, bedarf für die meisten Schülerinnen und Schüler keiner zusätzlichen Anschauungshilfen durch Analogiemodelle - zumindest beim Aufbau des Stromkreiskonzepts. Andere Untersuchungen bestätigen den Stellenwert der Lehrkraft und die Abhängigkeit erfolgreichen Unterrichts von deren fachdidaktischem Wissen bzw. der Unterrichtsqualität, so u. a. die PLUS-Studie (Lange et al. 2012). Allerdings sollte in nachfolgenden Studien diese Annahme empirisch genauer überprüft werden. Das ist insofern von besonderer Bedeutung, als im naturwissenschaftlichen Sachunterricht das erforder-

\footnotetext{
${ }^{1}$ Die Lehrerin hatte im Lehramtsstudium das Hauptfach Physik (einschließlich Didaktik der Physik) studiert.
} 
liche fachdidaktische Wissen und Fachwissen meist nicht in ausreichendem Maße vorliegen, da nur ein sehr geringer Teil der Grundschullehrerinnen und -lehrer naturwissenschaftliche Fächer (insbesondere Chemie und Physik) und ihre Didaktiken studiert hat (Schmidt 2015).

Im Unterschied zum Aufbau des Stromkreiskonzepts führte der Einsatz von verschiedenen Modellen beim Aufbau korrekter Stromverbrauchsvorstellungen zu unterschiedlichen langfristigen Lernentwicklungen in den Gruppen (VS1, VS2, VG). Der Einsatz des Riemenmodells brachte hierbei einen höheren Erfolg im Follow-up Test beim Aufbau korrekter Vorstellungen als der Einsatz des Wassermodells bzw. als der Unterricht ohne Analogiemodelle (VG). Eine Erklärung für diesen Befund liefert die Structure Mapping Theorie (Gentner 1983). Gemäß dieser Theorie ist es für erfolgreiches Lernen wichtig, dass wesentliche Elemente und deren Beziehungen zwischen den Repräsentationen identifiziert und Bezüge hergestellt werden können (ebd.). Diesbezüglich hat das Riemenmodell Vorteile: Es bildet gut nachvollziehbar sowohl Oberflächenmerkmale (Entsprechungen von Bauteilen bei Stromkreis und Modell) als auch strukturelle Merkmale (Kreisfluss; kein „Verbrauch“von Teilchen) ab. Die Bezüge zwischen dem sekundären (Modell) und dem primären Lerngegenstand (Stromkreis) konnten daher sehr gut erkannt und nachvollzogen werden. Hinzu kommt der Faktor der konkreten Handlung beim Nachvollziehen der Energiequelle im Riemenmodell: Durch eigenhändiges Kurbeln wird der Propeller in Bewegung gesetzt. Beim Doppelwassersäulenmodell hingegen wird das Rädchen durch die potentielle Energie in den Wassersäulen angetrieben, was einen deutlich höheren Abstraktionsanspruch darstellt und für viele Schülerinnen und Schüler der dritten Jahrgangsstufe eine Überforderung darstellen könnte. Es kann vermutet werden, dass die Energie, die bei der Doppelwassersäule in Form von potentieller Energie in den Säulen steckt, für die Kinder schwieriger nachvollziehbar ist als die Energie, die bei einer Kurbel aktiv handelnd in Form von Anstrengung erfahren wird. Deshalb ist die Tiefenstruktur des Analogiemodells (hier: Erhalt von Elektronen, Energieumwandlung) beim Wassermodell weniger gut erkennbar. Dies könnte dazu beigetragen haben, dass das Verständnis der strukturellen Komponente des Lerngegenstandes (Abbau von Stromverbrauchsvorstellungen) geringer ausfällt. Das Wassermodell ist zudem komplexer und veranschaulicht sowohl den Stromfluss als auch die Spannung. Der oben beschriebene Zuschnitt dieses Modells (vgl. Abschn. 2.3) könnte manchen Schülerinnen und Schülern eine geringere Lernhilfe sein. Die beschriebenen Annahmen müssten jedoch in weiteren Untersuchungen, die die Lernprozesse der Kinder genauer in den Blick nehmen, überprüft werden. Dabei gilt es insbesondere zu prüfen, inwieweit Schwierigkeiten im Verständnis des Stromverbrauchskonzepts durch das Wassermodell selbst erzeugt werden. In der vorliegenden Studie sowie der Studie von Haider (2010) haben die Schülerinnen und Schüler, die mit den Wassermodellen gearbeitet haben, geringere Lernerfolge erzielt als diejenigen, die die Riemenmodelle genutzt haben. Da Wasser-Analogiemodelle in weiterführenden Schulen zum Thema Strom häufig eingesetzt werden (z. B. Hampl et al. 2010; Meyer und Schmidt 2010; Scharf 2010), sollte in nachfolgenden Studien differenzierter untersucht werden, worin die Lernschwierigkeiten bestehen und wie sie ggf. kompensiert werden können. Hierzu müssen die Lern- und Verstehensprozesse in den Blick genommen werden, beispielsweise durch Metho- 
den des „lauten Denkens“. Das Doppelwassersäulenmodell repräsentiert neben dem Stromfluss auch die Spannung, was aus physikalischer Sicht für das weiterführende Lernen relevant ist (Burde und Wilhelm 2016b). So bliebe zu überprüfen, ob ein Einsatz von Wassermodellen in der Grundschule die Anschlussfähigkeit an Lerninhalte der Sekundarstufe (z. B. bzgl. des Elektronengasmodells, Burde und Wilhelm 2016b) eher unterstützt als der Einsatz anderer Modelle.

Unerwartete Ergebnisse erbrachten die differentiellen Analysen (Hypothese 5): Es zeigte sich, dass die Analogiemodelle für Schülerinnen und Schüler mit ungünstigen Lernvoraussetzungen keine zusätzlichen Veranschaulichungshilfen für das Verständnis des Stromkreiskonzeptes und für den Abbau von Stromverbrauchsvorstellungen darstellten. Andererseits wurde aber auch deutlich, dass die Schülerinnen und Schüler mit ungünstigen Lernvoraussetzungen die Analogiemodelle in gleichem Maße nutzen konnten wie die anderen Schülergruppen. Mit dem Modelleinsatz wurde entsprechend keine Schülergruppe benachteiligt.

Weiterführend wäre zu untersuchen, wie stabil das vor allem über Analogiemodelle aufgebaute konzeptuelle Wissen zum Stromfluss und -verbrauch ist, da Analogiemodelle keine eigenen Evidenzen zur Korrektheit liefern. Die Modelle unterstützen als didaktische Maßnahme oder Veranschaulichungshilfe die Verständlichkeit des Lerninhalts, sie erzeugen aber keine neuen Erkenntnisse. Hier könnten Untersuchungen, in denen verschiedene Anwendungskontexte verwendet werden und in denen ein naher und ferner Transfer geprüft wird, relevante Ergebnisse erbringen. Auch dies lässt sich mit Hilfe der Structure Mapping Theorie (Gentner 1983) begründen: Es konnte gezeigt werden, dass die Akzeptanz für Analogien bei Oberflächenähnlichkeiten höher ist (Blanchette und Dunbar 2001), dass eine tiefere Verarbeitung und damit größere Lernerfolge jedoch bei einer höheren Ähnlichkeit auf der Tiefenstrukturebene erzielt werden (Kurtz et al. 2001). Diese Befunde müssten jedoch für das naturwissenschaftliche Lernen im Sachunterricht der Grundschule überprüft werden. Auch sollte an weiteren Phänomenen bzw. Inhaltsbereichen untersucht werden, inwieweit durch einen Unterricht, der mit Hilfe von Analogien auf verschiedene Oberflächen- und Tiefenstrukturmerkmale fokussiert, entsprechende Ergebnisse erzielt werden. Angelehnt an die hier vorliegenden Befunde und die Untersuchungen von Lohrmann et al. (2014, 2018) könnten dann allgemeinere Regelhaftigkeiten abgeleitet werden, wodurch die Theorie Gentners (1983) auch für den naturwissenschaftlichen Sachunterricht der Grundschule eine größere Relevanz erhielte.

Förderung Das Projekt wurde von der DFG unter der Nummer HA 6072/2-1 gefördert

Funding Open Access funding provided by Projekt DEAL.

Open Access Dieser Artikel wird unter der Creative Commons Namensnennung 4.0 International Lizenz veröffentlicht, welche die Nutzung, Vervielfältigung, Bearbeitung, Verbreitung und Wiedergabe in jeglichem Medium und Format erlaubt, sofern Sie den/die ursprünglichen Autor(en) und die Quelle ordnungsgemäß nennen, einen Link zur Creative Commons Lizenz beifügen und angeben, ob Änderungen vorgenommen wurden.

Die in diesem Artikel enthaltenen Bilder und sonstiges Drittmaterial unterliegen ebenfalls der genannten Creative Commons Lizenz, sofern sich aus der Abbildungslegende nichts anderes ergibt. Sofern das betreffende Material nicht unter der genannten Creative Commons Lizenz steht und die betreffende Handlung 
nicht nach gesetzlichen Vorschriften erlaubt ist, ist für die oben aufgeführten Weiterverwendungen des Materials die Einwilligung des jeweiligen Rechteinhabers einzuholen.

Weitere Details zur Lizenz entnehmen Sie bitte der Lizenzinformation auf http://creativecommons.org/ licenses/by/4.0/deed.de.

\section{Literatur}

Blanchette, I., \& Dunbar, K. (2001). Analogy use in naturalistic settings: the influence of audience, emotion, and goals. Memory \& Cognition, 29(5), 730-735.

Burde, J.-P., \& Wilhelm, T. (2016a). Moment mal ... (22): Hilft die Wasserkreislaufanalogie? Praxis der Naturwissenschaften - Physik in der Schule, 65(1), 46-49.

Burde, J.-P., \& Wilhelm, T. (2016b). Die Elektrizitätslehre mit dem Elektronengasmodell. Praxis der Naturwissenschaften - Physik in der Schule, 65(8), 18-24.

Carey, S. (1985). Conceptual change in childhood. Cambridge, MA: MIT Press.

Chi, M. T., Feltovich, P. J., \& Glaser, R. (1981). Categorization and representation of physics problems by experts and novices. Cognitive Science, 5, 121-152.

De Groot, A.D., \& Gobet, F. (1996). Perception and memory in chess. Assen: Van Gorcum.

Dochy, F., de Rijdt, C., \& Dyck, W. (2002). Cognitive prerequisites and learning: How far have we progressed since Bloom? Implications for educational practice and teaching. Active Learning in Higher Education, 3(3), 265-284.

Dudeck, W.-G. (1997). Analyse von Denkprozessen in einem analogieorientierten Elektrizitätslehreunterricht - Fallstudie in einer 10. Gymnasialklasse. Aachen: Verlag Mainz.

Duit, R. (2011). Schülervorstellungen und Lernen von Physik - Stand der Dinge und Ausblick. In R. Müller, R. Wodzinski \& M. Hopf (Hrsg.), Physik allgemein (S. 1-5). Köln: Aulis.

Duit, R., \& Glynn, S. M. (1995). Learning science in the schools: research reforming practice. Mahwah: Lawrence Erlbaum.

Ferguson, R. W. (1994). MAGI. Analogy-based encoding using symmetry and regularity. In Proceedings of the Sixteenth Annual Conference of the Cognitive Science Society. Hillsdale, NJ: Erlbaum.

Gentner, D. (1983). Structure-mapping. A theoretical framework for analogy. Cognitive Science, 7(2), 155-170.

Gentner, D., Loewenstein, J., \& Thompson, L. (2003). Learning and transfer: a general role for analogical encoding. Journal of Educational Psychology, 95(2), 393-408.

Gentner, D., Loewenstein, J., Thompson, L., \& Forbus, K. D. (2009). Reviving inert knowledge: analogical abstraction supports relational retrieval of past events. Cognitive Science, 33, 1343-1382.

Haider, M. (2010). Der Stellenwert von Analogien für den Erwerb naturwissenschaftlicher Erkenntnisse. Bad Heilbrunn: Klinkhardt.

Hampl, U., Heepmann, B., Rossa, E., Schröder, W., Sinterhauf, R., \& Wisniewski, H. (Hrsg.). (2010). Natur und Technik. Neue Ausgabe. Physik/ Chemie/ Biologie 7. Schülerbuch. Hauptschule Bayern. Berlin: Cornelsen.

Hardy, I. (2012). Kognitive Strukturierung in der Grundschule - Empirische Zugänge zu einem heterogenen Konstrukt der Unterrichtsforschung. In F. Hellmich, S. Förster \& F. Hoya (Hrsg.), Bedingungen des Lehrens und Lernens in der Grundschule. Bilanz und Perspektiven (S. 51-64). Wiesbaden: VS.

Hesse, F. W. (1991). Analoges Problemlösen: eine Analyse kognitiver Prozesse beim analogen Problemlösen. Weinheim: Psychologie-Verlags Union.

Jonen, A., Möller, K., \& Hardy, I. (2003). Lernen als Veränderung von Konzepten - am Beispiel einer Untersuchung zum naturwissenschaftlichen Lernen in der Grundschule. In D. Czech \& H. J. Schwier (Hrsg.), Lernwege und Aneignungsformen im Sachunterricht (S. 93-108). Bad Heilbrunn: Klinkhardt.

Kircher, E. (1989). Analogien im Physikunterricht. In W. Schneider (Hrsg.), Wege in der Physikdidaktik (S. 47-57). Erlangen: Palm und Enke.

Kircher, E. (1995). Analogien im Sachunterricht der Primarstufe. Sachunterricht und Mathematik in der Primarstufe, 23(5), 192-197.

Kircher, E., Girwidz, R., \& Häußler, P. (Hrsg.). (2009). Physikdidaktik. Theorie und Praxis. Berlin, Heidelberg: Springer.

Kurtz, K. J., Miao, C.-H., \& Gentner, D. (2001). Learning by analogical bootstrapping. Journal of the Learning Sciences, 10(4), 417-446.

Lange, K., Kleickmann, T., \& Möller, K. (2012). Die Bedeutung des fachdidaktischen Wissens von Lehrkräften für Lernfortschritte von Schülerinnen und Schülern im Sachunterricht der Grundschule. In 
H. Bayrhuber, U. Harms, B. Muszynski, B. Ralle, M. Rothgangel, L.H. Schön, L.H. Vollmer \& H. G. Weigang (Hrsg.), Formate fachdidaktischer Forschung: Empirische Projekte - historische Analysen - theoretische Grundlegungen (S. 315-334). Münster: Waxmann.

Lenzner, A. (2009). Visuelle Wissenskommunikation: Effekte von Bildern beim Lernen. Kognitive, affektive und motivationale Effekte. Hamburg: Dr. Kovac.

Lohrmann, K., \& Hartinger, A. (2012). Kindliche Präkonzepte im Sachunterricht. Empirische Forschung und praktischer Nutzen. Die Grundschulzeitschrift, 26(252/253), 16-21.

Lohrmann, K., Groß Ophoff, J., \& Hartinger, A. (2018). Analoges Enkodieren und die Fähigkeit zum Transfer im naturwissenschaftlichen Sachunterricht. Zeitschrift für Grundschulforschung, 11(2), 365-381.

Lohrmann, K., Hartinger, A., Schwelle, V., \& Hartig, J. (2014). Die Bedeutung der (Un-)Ähnlichkeit von Beispielen für den Aufbau von konzeptuellem Wissen. Zeitschrift für Grundschulforschung, 6(2), 60-73.

Mayer, R. E., Heiser, J., \& Lonn, S. (2001). Cognitive constraints on multimedia learning: when presenting more material results in less understanding. Journal of educational psychology, 93(1), 187.

Meyer, L., \& Schmidt, G.-D. (2010). Physik 8 Lehrbuch Gymnasium Bayern. Berlin: Duden Verlag.

Ministerium für Schule und Weiterbildung des Landes Nordrhein-Westfalen (2012). Richtlinien und Lehrpläne für die Grundschule. Frechen: Ritterbach.

Möller, J., \& Müller-Kalthoff, T. (2000). Lernen mit Hypertext. Effekte von Navigationshilfen und Vorwissen. Zeitschrift für Pädagogische Psychologie, 14(2/3), 116-123.

Möller, K., Jonen, A., Hardy, I., \& Stern, E. (2002). Die Förderung von naturwissenschaftlichem Verständnis bei Grundschulkindern durch Strukturierung der Lernumgebung. In M. Prenzel \& J. Doll (Hrsg.), Bildungsqualität von Schule: Schulische und außerschulische Bedingungen mathematischer, naturwissenschaftlicher und überfachlicher Kompetenzen (S. 176-191). Weinheim: Beltz.

Muckenfuß, H. (1995). Lernen im sinnstiftenden Kontext. Entwurf einer zeitgenössischen Didaktik des Physikunterrichts. Berlin: Cornelsen.

Niedderer, H., \& Gohmert, B. (1982). Zur Einführung des Spannungsbegriffs. Naturwissenschaften im Unterricht. Physik, Chemie, 30(11), 394-399.

Posner, G. J., Strike, K. A., Hewson, P. W., \& Gertzog, W. A. (1982). Accomodation of a scientific conception: toward a theory of conceptual change. Science Education, 66(2), 211-227.

v. Rhöneck, C., \& Völker, B. (1982). Einfache Elektrizitätslehre zwischen physikalischem Anspruch und Lernschwierigkeiten. Naturwissenschaften im Unterricht P/C, 30(11), 406-412.

Rittle-Johnson, B., \& Star, J.R. (2009). Compared with what? The effects of different comparisons on conceptual knowledge and procedural flexibility for equation solving. Journal of Educational Psychology, 101(3), 529-544.

Ruppert, M. (2017). Wege der Analogiebildung - Eine qualitative Studie über den Prozess der Analogiebildung beim Lösen von Aufgaben. Münster: WTM.

Sagi, E., Gentner, D., \& Lovett, A. (2012). What difference reveals about similarity. Cognitive Science, 36(6), 1019-1050.

Scharf, K.-H. (Hrsg.). (2010). Natur Plus. Physik/Chemie/Biologie 7. Jahrgangsstufe. Braunschweig: Schroedel.

Schmidt, M. (2015). Professionswissen von Sachunterrichtslehrkräften. Zusammenhangsanalyse zur Wirkung von Ausbildungshintergrund und Unterrichtserfahrung auf das fachspezifische Professionswissen im Unterrichtsinhalt „, Verbrennung “. Berlin: Logos.

Schwedes, H. (1985). The importance of water circuits in teaching electric circuits. In R. Duit, W. Jung \& C. v. Rhöneck (Hrsg.), Aspects of understanding electricity (S. 319-330). Kiel: IPN.

Spreckelsen, K. (1997). Phänomenkreise als Verstehenshilfe. In W. Köhnlein, B. Marquart-Mau \& H. Schreier (Hrsg.), Kinder auf dem Weg zum Verstehen der Welt (S. 111-127). Bad Heilbrunn: Klinkhardt.

Staatsinstitut für Schulqualität und Bildungsforschung (ISB) (2017). LehrplanPlus, Grundschule. München: Maiss.

Sternberg, R. J. (1977). Intelligence, information processing, and analogical reasoning. Hillsdale, New Jersey: Lawrence Erlbaum.

Treagust, D.F., \& Duit, R. (2008). Conceptual change: a discussion of theoretical, methodological and practical challenges for science education. Cultural Studies of Science Education, 3(2), 297-328.

Vosniadou, S. (1994). Capturing and modelling the process of conceptual change. Learning and Instruction, 4(1), 45-69.

Vosniadou, S. (2001). How children learn. Brüssel: International Academy of Education. 
Vosniadou, S. (2007). Re-framing the conceptual change approach in learning and instruction. Amsterdam u. a.: Elsevier.

Vosniadou, S., \& Brewer, W. (1987). Theories of knowledge restructuring in development. Review of Educational Research, 57(1), 51-67.

Wagenschein, M. (2010). Verstehen lehren. Genetisch-Sokratisch-Exemplarisch. Weinheim und Basel: Beltz.

Wiesner, H. (1995). Untersuchungen zu Lernschwierigkeiten von Grundschülern in der Elektrizitätslehre. Sachunterricht und Mathematik in der Primarstufe, 23(2), 50-58.

Wilhelm, T. (2015). Moment mal...(18): Elektronen als Energieträger? Naturwissenschaften Physik in der Schule 64(6), 47-49. 